\title{
PASOS INFERIORES AL FERROCARRIL: AVANZANDO CON EL EMPUJE OLEODINÁMICO
}

\author{
(RAILWAY CROSSINGS: DRIVING THE STRUCTURE UNDER THE RAILWAY BY MEANS \\ OF OLEODINAMIC JACKS)
}

Ramón Escribano Méndez ${ }^{1}$, Ingeniero de Caminos

Rafael López Palomar², Ingeniero de Caminos

Andrés J. Ruiz Viedma ${ }^{3}$, Ingeniero de Caminos MADRID/ESPAÑA

\section{RESUMEN}

"No hay mejor paso a nivel que el paso muerto", suelen decir los encargados del tema. Pero, hasta hace muy poco, la eliminación de estos conflictivos cruces no sólo representaba mucho dinero, sino una larga obra, con la consabida secuela de precauciones, limitaciones de velocidad y problemas para el tráfico ferroviario. Sin embargo, un novedoso sistema, basado en clavar mediante gatos hidráulicos la estructura de hormigón del paso a diferente nivel en el terraplén de la via, permite hacer en pocas semanas lo que antes se tardaba largos meses. La aplicación de este nuevo método reviste gran importancia, especialmente si se piensa que las lineas a $200 \mathrm{~km} / \mathrm{h}$ prácticamente no pueden tener cruzamientos al mismo nivel.

\section{SUMMARY}

"The best level crossing is a dead crossing", those involved are accustomed to say. Yet, until a short time ago, eliminating these conflictive crossings not only implied a great deal of money, but prolonged building work, with the all too familiar sequel of precautions, speed restrictions and problems for rail traffic. However, a brand new system, based on using hydraulic jacks to drive the concrete structure of the crossing to a different level in the track embankment, allows the execution in a few weeks of what before took long months. Use of this new method acquires great importance, especially if one considers that $200 \mathrm{~km} /$ hour tracks virtually cannot have crossings at the same level.

\section{A MODO DE RECORDATORIO}

La técnica del empuje oleodinámico de estructuras aplicada a la construcción de pasos inferiores al ferrocarril ya se ha descrito, suficientemente, en diversos articulos publicados en diferentes revistas de divulgación técnica. "Informes de la Construcción" dedicó en su n. 403 de Septiembre/Octubre de 1989 un amplio espacio al desarrollo de este procedimiento en una obra concreta, analizando todos los aspectos y ventajas, tanto técnicas como económicas, y destacando la importante disminución en la afección al tráfico ferroviario respecto de los procedimientos tradicionales.

A modo de recordatorio, extractaremos a continuación las fases y elementos que intervienen en la cons.

1) Gerente de Pasos a Nivel de RENFE.

2) Jefe del Plan de Supresión de Pasos a Nivel de RENFE.

3) Director de atros ESPAÑA. S. A trucción de un paso inferior por empuje oleodinámico:

1. Trabajos preliminares y obras auxiliares:

- Excavaciones previas y desvio de servicios afectados.

- Construcción de la plataforma de deslizamiento, muretes guia y muro de reacción, todos en hormigón armado.

- Colocación de lámina de polietileno sobre la plataforma de deslizamiento.

\section{Construcción de la estructura:}

- Se realiza en hormigón armado_con sección transversal de marco y sobre la plataforma de deslizamiento. 
- Los muros se prolongan con dos grandes ménsulas o cuchillos en forma de triángulo rectán. gulo invertido, que contienen el terreno durante la traslación. El borde frontal biselado facilita la excavación y el corte del terreno sobrante.

- Sobre el frente de avance se construyen una o más vigas de sustentación, que arriostran los cuchillos y permiten el apoyo de las vigas de maniobra en las primeras fases de traslación.

- En la parte posterior se adopta la forma de las aletas que, una vez situada la estructura en su posición definitiva, se prolongan para formar el acceso al paso inferior.

\section{Apeo y sustentación de la vía:}

- La vía se refuerza longitudinalmente con un apeo formado por fajos de carriles empaquetados con abrazaderas especiales tipo Garruti.

- Transversalmente se colocan bajo las vías unas vigas de acero que llamanos "vigas de manio. bra". Éstas se apoyan en la estructura y, al otro lado de las vias, sobre el terreno.

\section{Traslación de la estructura}

- Se realiza mediante un conjunto de gatos hidráulicos movidos por una central, los cuales se apoyan sobre el muro de reacción directamente al comienzo de la traslación, y mediante prolongadores metálicos en las fases sucesivas.

- La excavación del terreno se realiza previamente a cada fase de empuje, con un avance determinado por la estabilidad del frente de ex. cavación, en función de las características del terreno.

- La estructura se desliza bajo las vigas de maniobra que sujetan la vía, las cuales transmiten las cargas de ferrocarril sobre la losa por medio de rodillos.

5. Trabajos complementarios:

- Cuando la estructura está en su posición definitiva, se retiran gradualmente las vigas de maniobra, se repone la banqueta de via sobre la losa y se retira el apeo.

- En la parte frontal de la estructura, si es preciso para la construcción de las aletas, se reali- za la demolición parcial de los cuchillos de avance, completándose las aletas y los muros de acompañamiento.

\section{INTRODUCCIÓN}

Desde que en 1987 se construyó en España el primer paso inferior por este sistema, que fue objeto del citado artículo, se han ejecutado aproximadamente 20 obras más por empuje oleodinámico y existen otras 12 en estudio.

La experiencia acumulada en esta serie de obras ha permitido constatar las ventajas del sistema que, aunque ya eran conocidas por su extensa aplicación en diversos paises europeos, han destacado notablemente en España, con la aportación de nuevas experiencias y la adaptación a nuestros condicionantes propios.

Destaca, sobre todo, la notable reducción en el tiempo de afección a la circulación de la vía afectada que, en término medio, se ha situado en la décima parte del necesario con los métodos tradicionales. Además, la mayor velocidad de circulación de las trenes en la zona de obra ( $30 \mathrm{~km} / \mathrm{h}$ respecto de $10 \mathrm{~km} / \mathrm{h}$ con métodos tradicionales) se traduce en una reducción considerable del tiempo perdido por los viajeros y mercancias, como consecuencia de la disminución de velocidad en el tramo de obra, y se reducen, también, las molestias y pérdidas de tiempo ocasionadas a los usuarios de la carretera afectada, ya que se adelanta su puesta en servicio definitivo.

Por otra parte, se obtienen otras ventajas derivadas del sistema constructivo, que pueden resumirse en:

- Eliminación de todos los trabajos que precisen corte de vía.

- Eliminación de trabajos nocturnos.

- Eliminación de toda actividad de maquinaria sobre la via y de los cortes de catenaria precisos.

- Disminución consecuente de interferencias con el tráfico ferroviario y total seguridad de circulación.

- Eliminación de problemas de cimentación.

- Facilidad de construcción de la estructura en espacio abierto.

- Control total de calidad de materiales y ejecución.

- Acabado de paramentos en hormigón visto, sin necesidad de revestimientos posteriores.

\section{VERSATILIDAD DEL SISTEMA (ADAPTACIÓN EN TRES DIMENSIONES)}

Los pasos construidos por el sistema te empuje oleodinámico se adaptan fácilmente a la libre y gálibo requeridos por el vial inferior, y al ancho de la plataforma necesaria para el vial superior. 


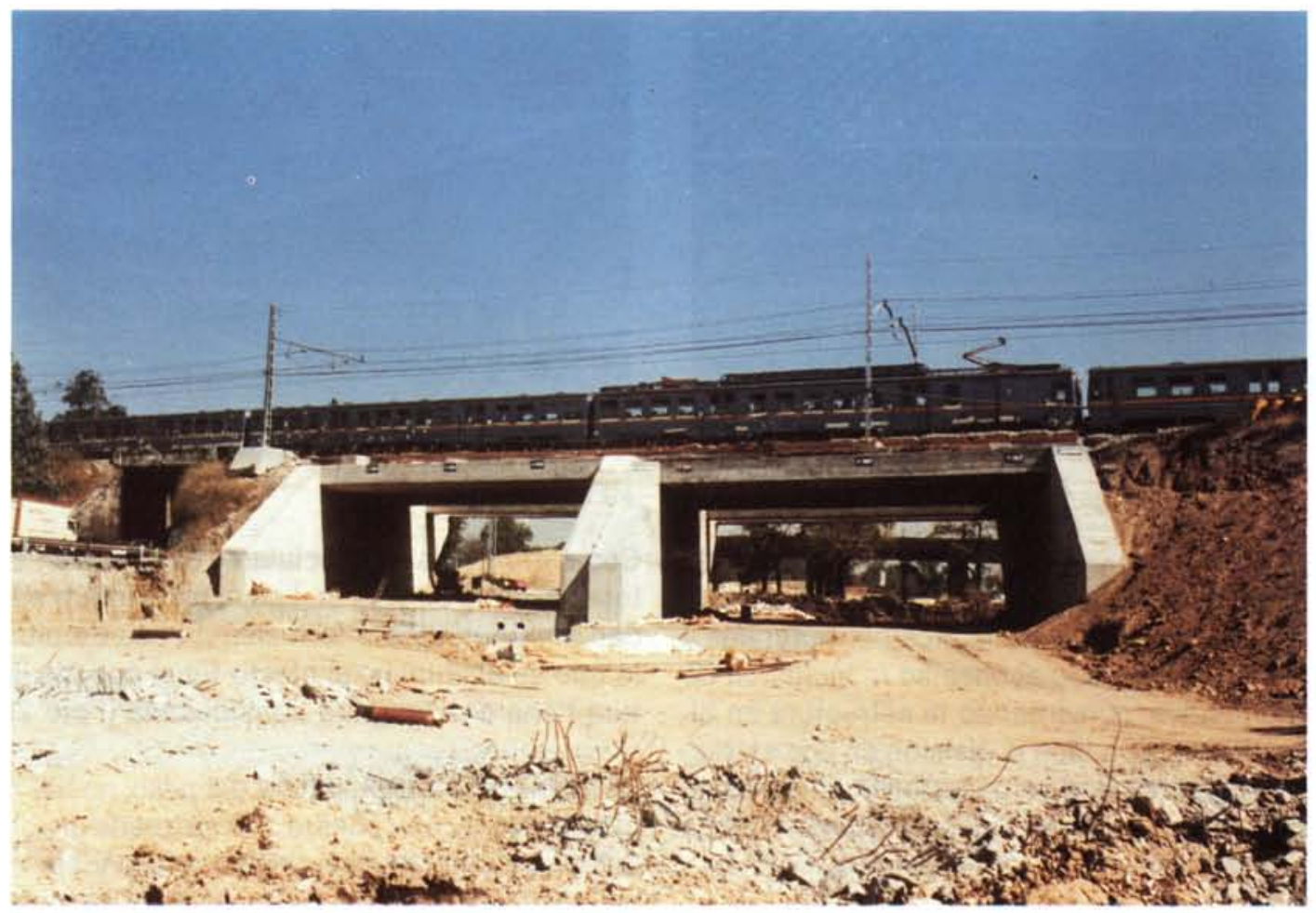

Foto 1.-Puente de los Franceses, Madrid.

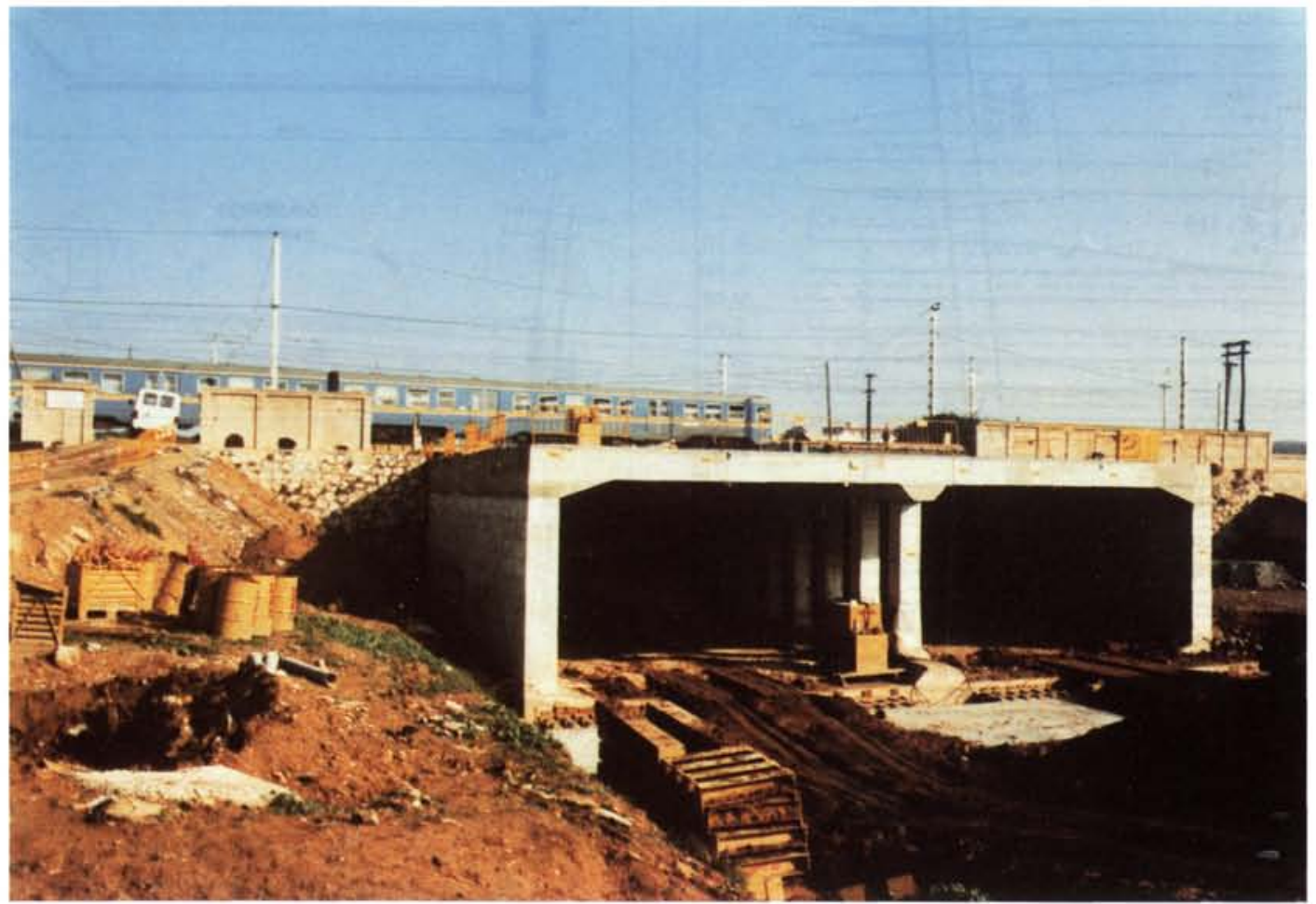

Foto 2.-Vilanova y la Geltrú, Barcelona. 

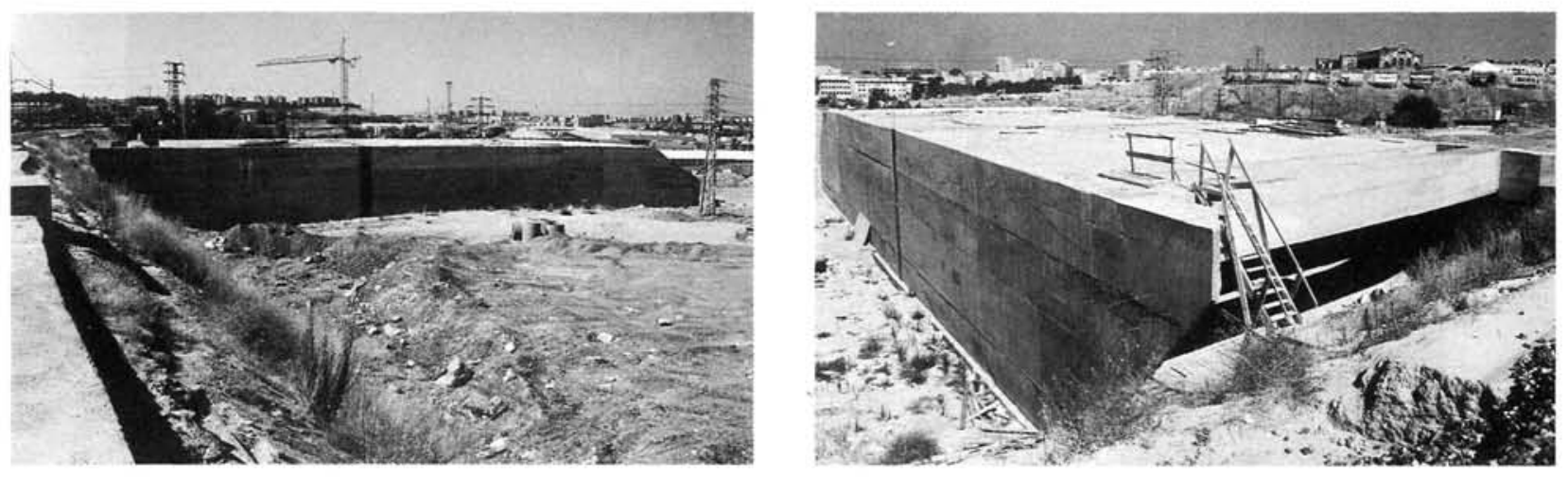

Fotos 3.- $a$ y b.

Con un solo vano se pueden alcanzar luces libres de $20 \mathrm{~m}$, e incluso superiores, aunque a partir de esta dimensión el espesor de los elementos estructurales y la complejidad del armado aconsejan la inclusión de apoyos intermedios, convirtiendo la estructura en bicelular o multicelular, o bien la división en dos estructuras independientes que se construyen en paralelo, comenzando la traslación de la segunda cuando se ha finalizado el emplazamiento de la primera (Foto 1).

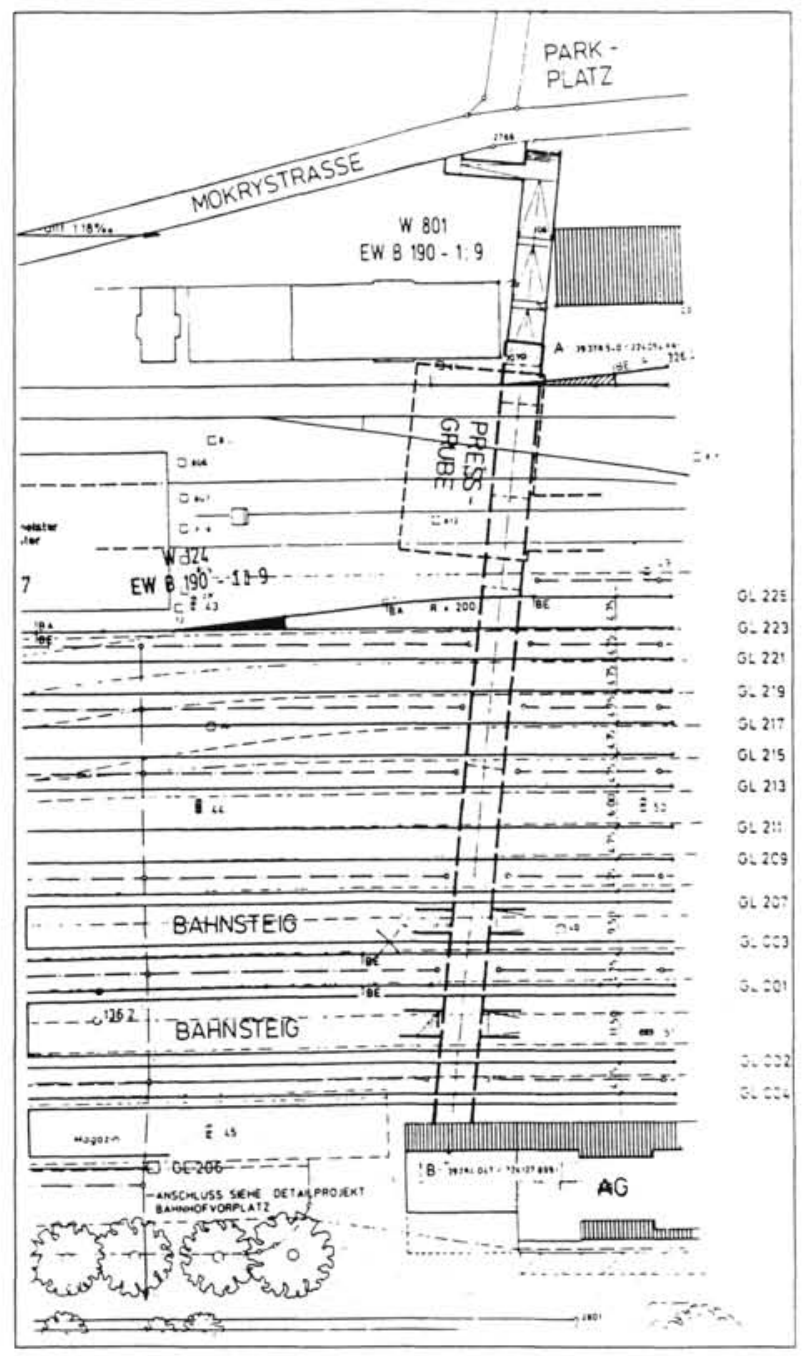

(C) Consejo Superior de Investigaciones Científicas Licencia Creative Commons 3.0 España (by-nc)
Con estructuras multicelulares se alcanzan anchuras totales, entre 20 y $40 \mathrm{~m}$. Los apoyos intermedios pueden ser continuos o formarse a base de pilares, con lo que se disminuye el efecto túnel cuando la estructura tiene una longitud considerable (Foto 2).

En cuanto a la longitud total del paso, no hay prácticamente restricción, puesto que se puede dividir en módulos que avanzan en sucesión, apoyándose cada uno en el anterior. De esta forma, se ha construido en España el Acceso Sur a Vallecas (Madrid), con una longitud total de 54,10 m (Fotos, 3 a y b), y en Austria (1984) se ha realizado uno de $80,50 \mathrm{~m}$ de longitud (ver esquemas). Con la división en módulos se pueden superar los 100 metros de longitud total, que en pocos casos serán necesarios.

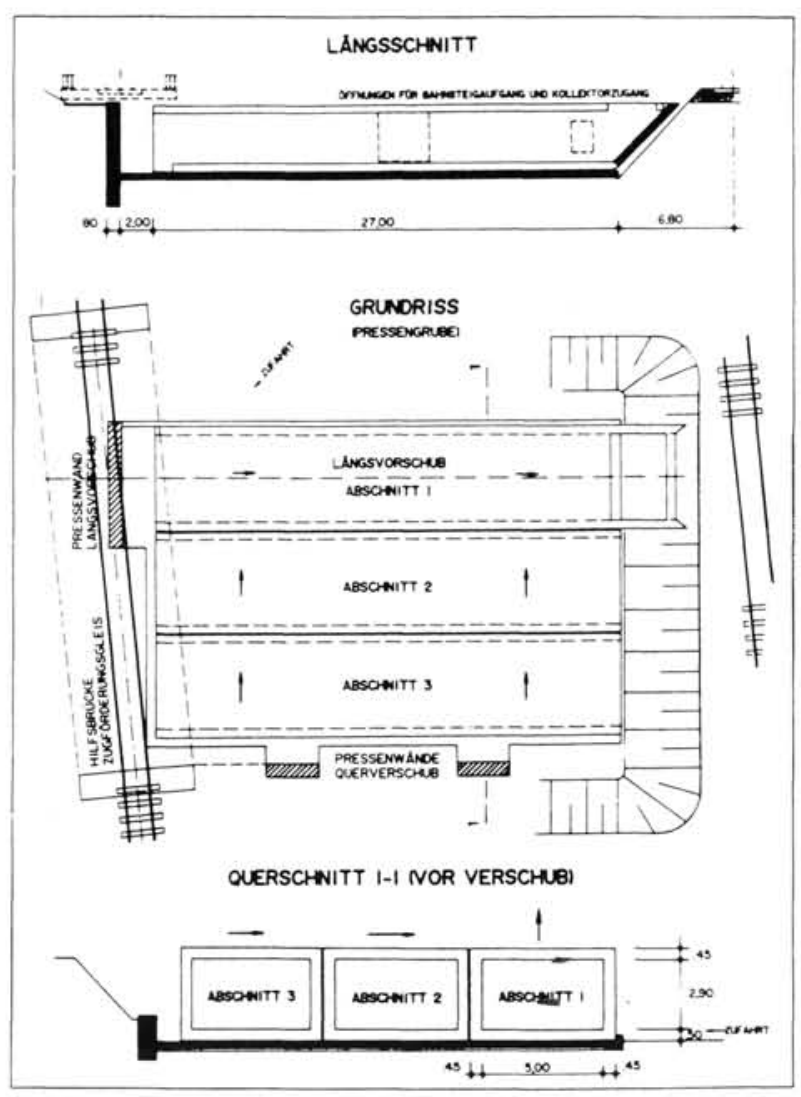

http://informesdelaconstruccion.revistas.csic.es 


\section{SALVANDO DIFICULTADES}

En el articulo publicado anteriormente se exponian las dificultades que representaban para el sistema el tipo de terreno a atravesar y la presencia de agua en el mis. mo.

En los tres pasos inferiores construidos en la linea Tarragona-San Vicente de Calders, por la Gerencia de Pasos a Nivel de RENFE, se ha obtenido una gran experiencia sobre la aplicación del sistema de empuje oleodinámico en condiciones adversas, llegándose a la conclusión de que el terreno no suele ser una condición limitativa para la construcción de pasos inferiores por este sistema, puesto que se pueden aplicar to. das las técnicas de excavación, consolidación o estabilización conocidas.

Las tres obras estaban proyectadas inicialmente para su ejecución por métodos convencionales, cambián. dose la solución al sistema de empuje oleodinámico tras el estudio realizado por ATROS ESPAÑA y partiendo de un informe geotécnico detallado. A continuación describiremos las dificultades planteadas en la ejecución de las tres obras mencionadas y los procedimientos aplicados para salvar dichas dificultades:

\section{Punto kilométrico $0 / 825$}

En este punto se ha construido un paso inferior dando acceso a la Playa del Milagro de Tarragona (Foto 4), con una luz libre de $12,00 \mathrm{~m}$ y un gálibo de $6,45 \mathrm{~m}$. La plataforma de via tiene una anchura de $23,00 \mathrm{~m}$, necesaria para la doble via de circulación principal y una via de apartadero con un cambio sobre la estructura.

En la fotografia 5 se aprecia la limitación en el espacio existente para la construcción de la estructura y obras auxiliares, debido a la presencia de edificaciones próximas y el paseo maritimo, en el que se debia mantener la circulación de vehiculos durante todo el tiempo de la obra. Estas circunstancias obligaron a diseñar y calcular la plataforma de deslizamiento, muro de reacción con unas caracteristicas distintas a las habituales en este tipo de obras, de forma que introduciendo elementos accesorios, se pudiera contar con la reacción necesaria para el empuje de la estructura utilizando el escaso espacio disponible.

En la fotografia 6 se observa que el terreno estaba constituido por un pedraplén o escollera, con elementos calizos de 30 a $50 \mathrm{~cm}$, sin material de relleno en los hue $\cos$ y apoyado sobre una base inclinada de roca cali$z a$. En estas condiciones, los avances se realizaron en tramos muy cortos, procurando contener lateralmente el pedraplén para no alterar la estabilidad del conjunto, ya que con la presión y el rozamiento producidos

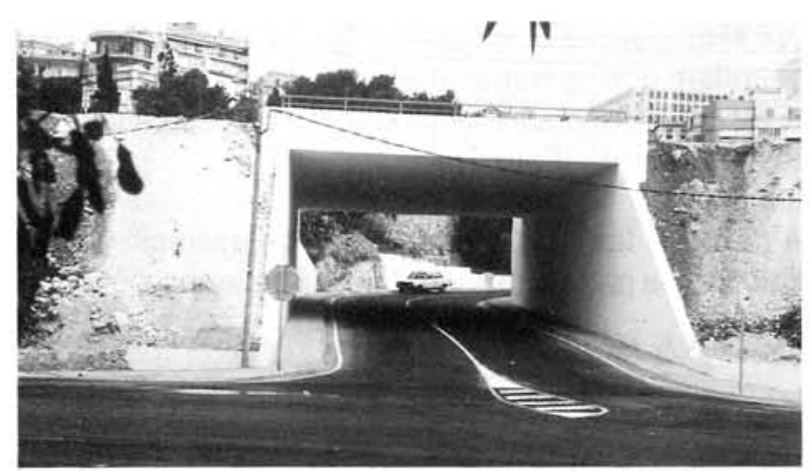

Foto 4

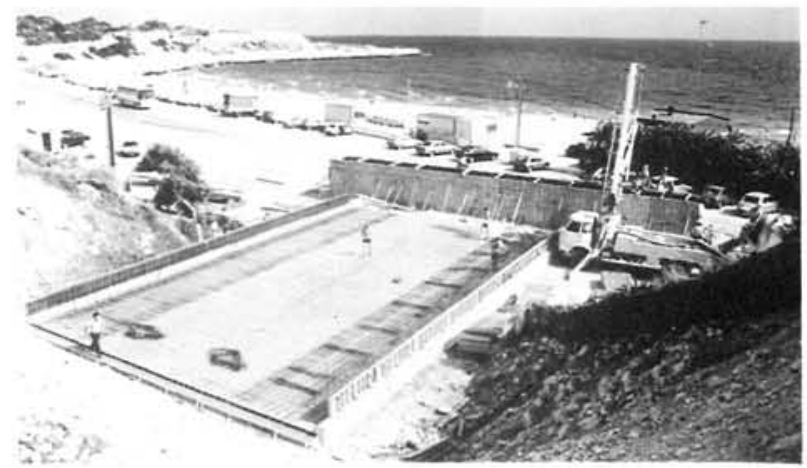

Foto 5

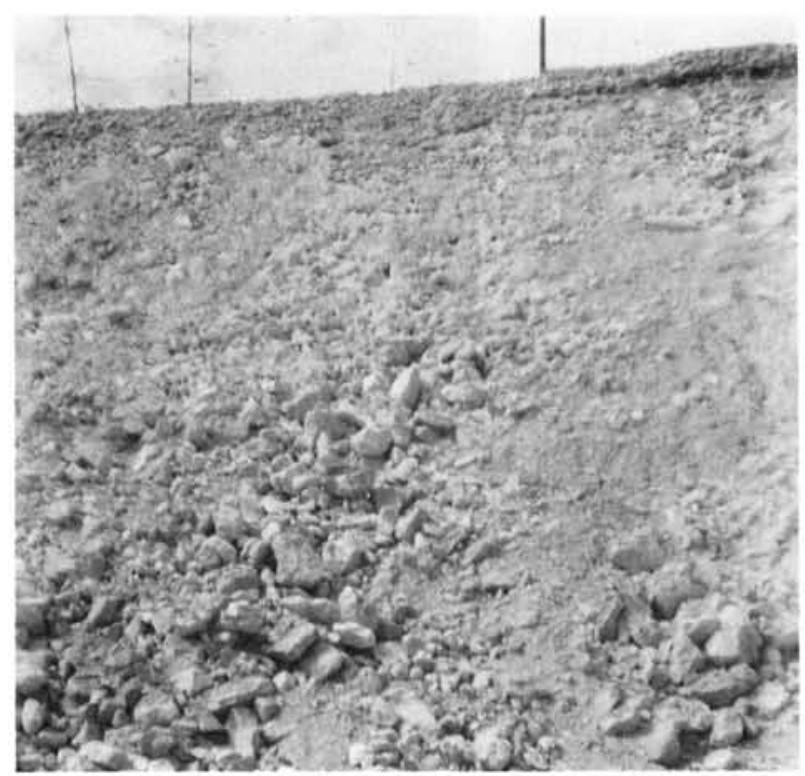

Foto 6

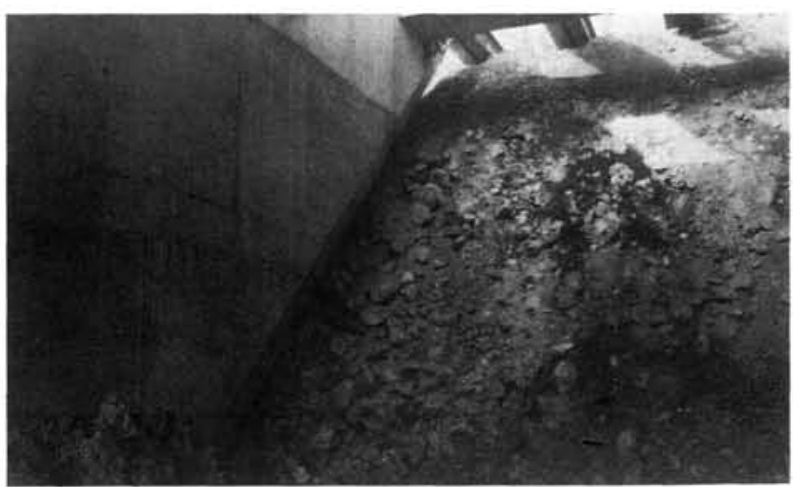

Foto 7

http://informesdelaconstruccion.revistas.csic.es 
por el empuje se originaban movimientos y giros en la escollera que se transmitian a otros más lejanos, con la amenaza constante de producirse un desprendimientos importante (Foto 7).

A partir de los $14,80 \mathrm{~m}$ de traslación apareció la roca caliza en la parte inferior del frente de avance, obligan- do a la utilización de martillos rompedores (Foto 8) para la excavación, haciéndola más lenta y dificultosa, como se puede apreciar en el diagrama de avance.

A pesar de las dificultades descritas, se invirtieron en el desplazamiento 11 dias para una traslación total de $24,00 \mathrm{~m}$, y con una media de avance de 2,18 m/dia. El

Foto 8

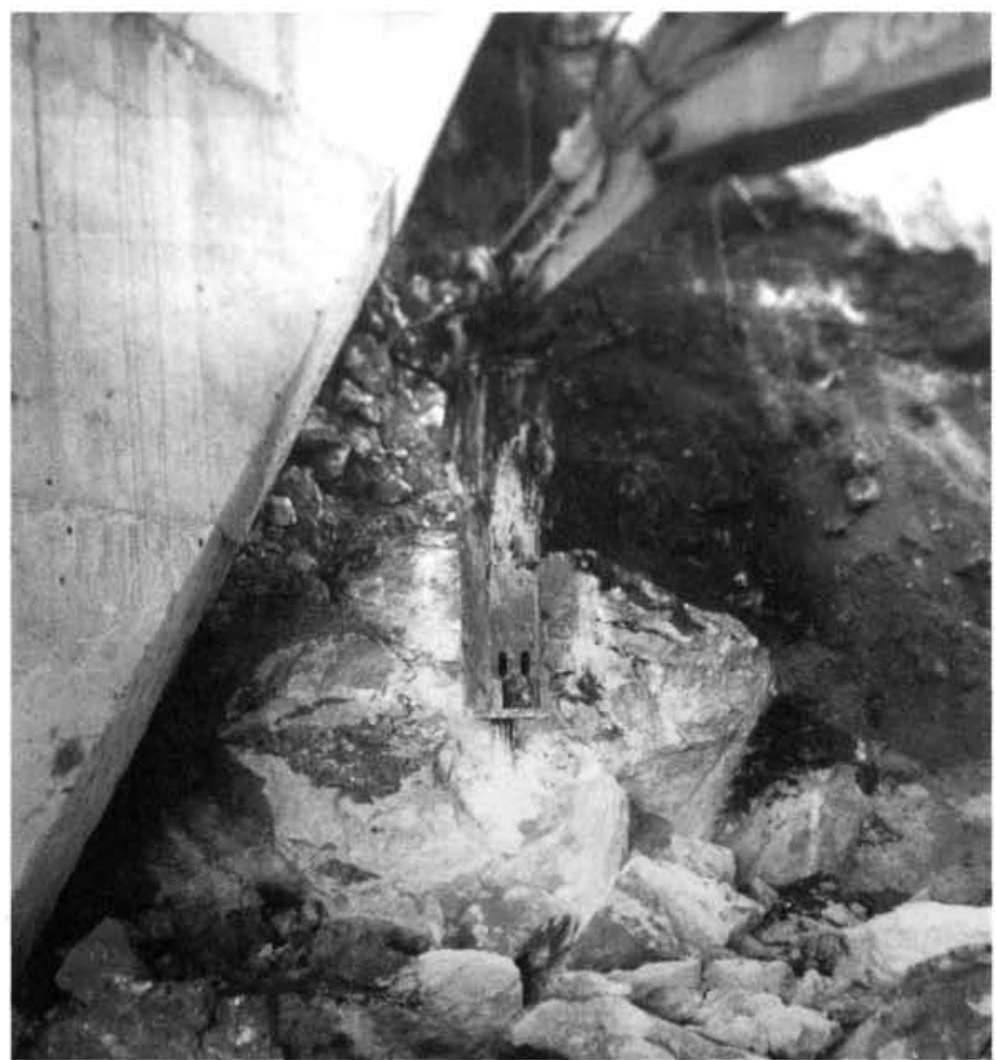

LINEA TARRAGONA - SAN VICENTE P. K. 0/825

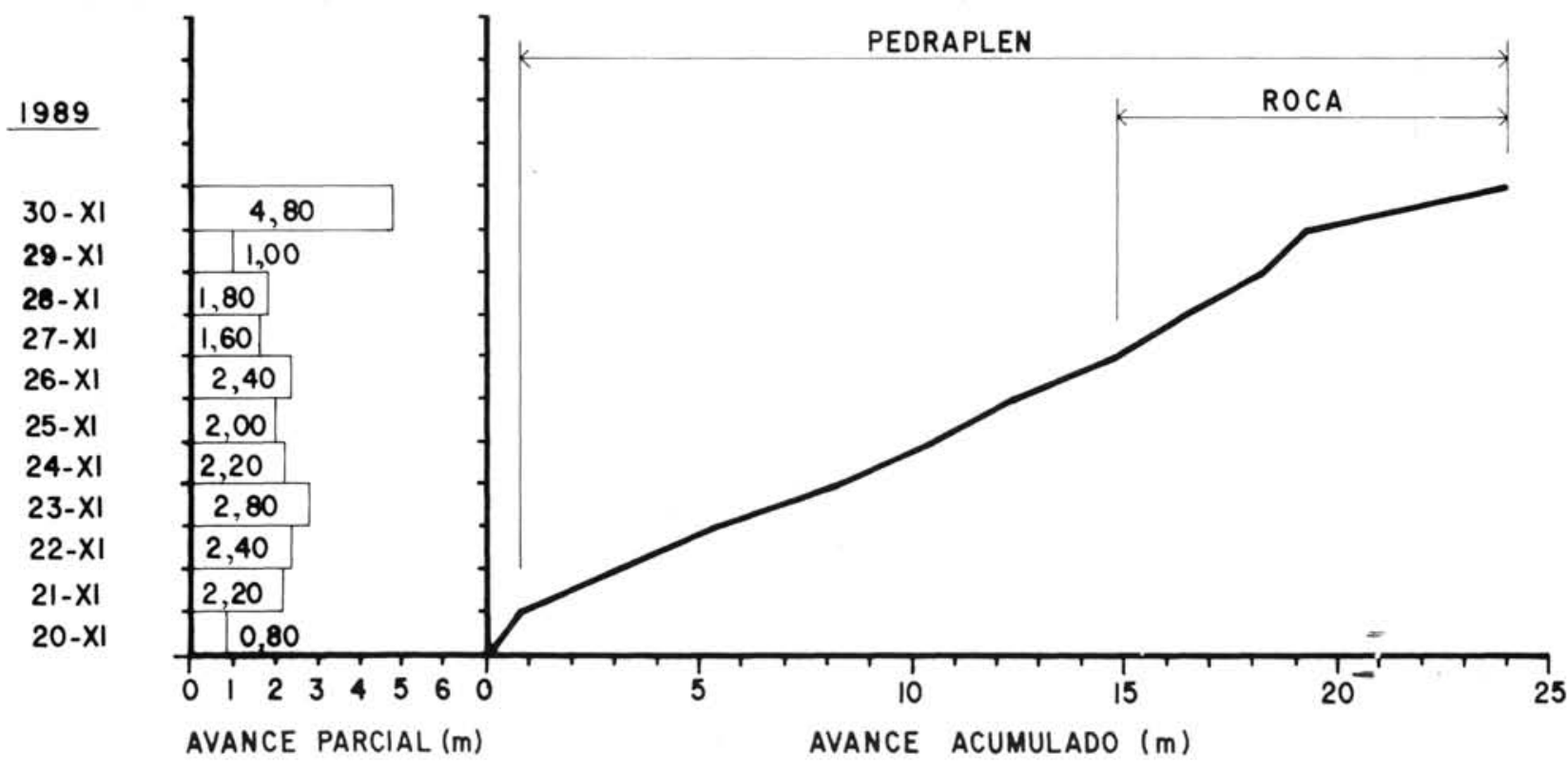


avance medio en la zona de pedraplén fue de 2,11 m, disminuyendo a 1,47 m/dia al aparecer la roca en la base. En el último dia, una vez que estaban sobrepasadas las dos vias, se realizaron $4,80 \mathrm{~m}$ de avance ayudado con excavación desde la zona opuesta al empuje.

Incluso con estas características del terreno se mantiene una notable diferencia en el tiempo de ejecución respecto de los métodos convencionales, cuya aplicación planteaba también grandes inconvenientes.

\section{Punto kilométrico $2 / 678$}

El paso inferior construido es de $9,00 \mathrm{~m}$ de luz y 4,25 metros de gálibo, dando acceso a la playa de La Savinosa desde la CN-340 (Foto 9). La plataforma es para doble via con un ancho total de 13,00 metros.

En este caso se repitieron las condiciones del terreno descritas en el anterior (pedraplén sobre roca); además, era preciso demoler simultáneamente una obra de paso antigua de fábrica de ladrillo con forma abovedada, con una anchura interior de 2,50 m (Foto 10).

La estructura se construyó en el lado mar, para lo cual fue necesario recrecer con tierras desde la cota de la playa hasta la base de la plataforma de deslizamiento, situada a una cota superior, y formar un gran terraplén sobre la misma playa para apoyo del muro de reacción (Foto 11).

La obra de fábrica existente se rellenó con tierra compactada para contrarrestar el posible efecto de un colapso inesperado de la bóveda durante la demolición, dejando alojado un tubo de hormigón para desagüe de pluviales durante el desplazamiento. En la zona opuesta a la estructura se terraplenó el espacio comprendido entre las aletas del paso antiguo para permitir el apoyo de las vigas de maniobra.

Tanto la excavación como el empuje se realizaron de la misma forma que en la obra anterior, con la dificultad añadida por la demolición de la obra de fábrica (Fo. to 12) y extremando las precauciones para no poner en peligro el servicio de la via en ningún momento.

El tiempo empleado en el empuje de la estructura fue de 10 dias, para una longitud total de traslación de 21,15 $\mathrm{m}$, obteniéndose un avance medio diario de 2,11 $\mathrm{m}$. En el diagrama de avance se aprecia la disminución del avance diario provocada por la demolición de la obra de fábrica antigua, con una media de 1,40 m/dia en esta fase. Terminada la demolición se aumentó considerablemente el rendimiento, realizándose $6,15 \mathrm{~m}$ de avance en el último dia, con excavación simultánea desde ambos lados de la via.

(c) Consejo Superior de Investigaciones Científicas Licencia Creative Commons 3.0 España (by-nc)

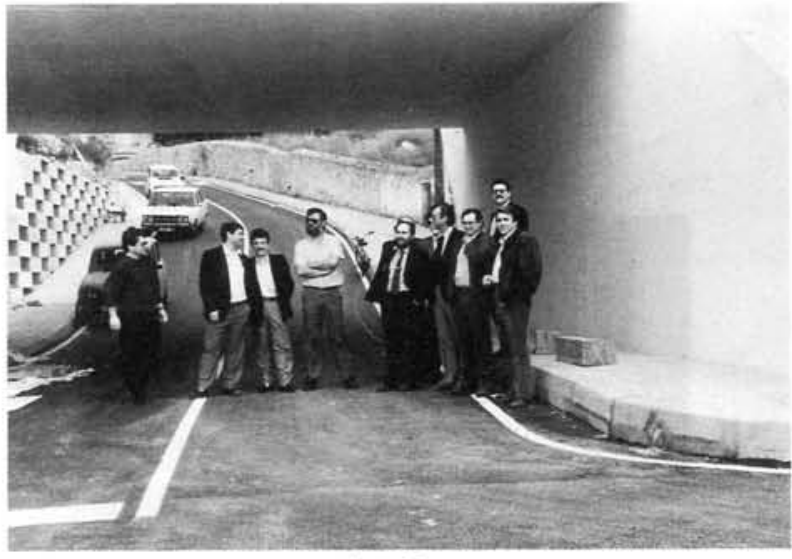

Foto 9
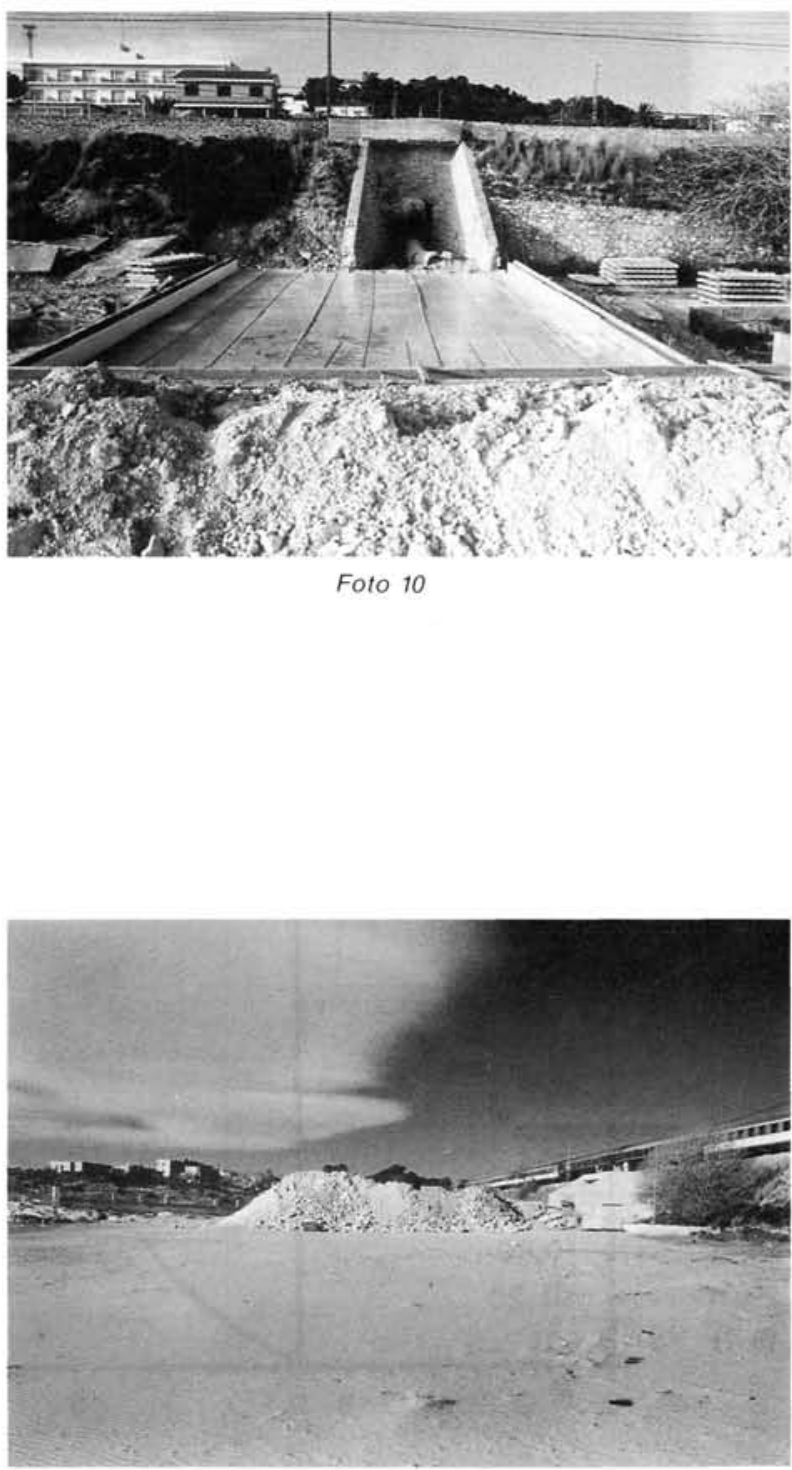

Foto 11

http://informesdelaconstruccion.revistas.csic.es 


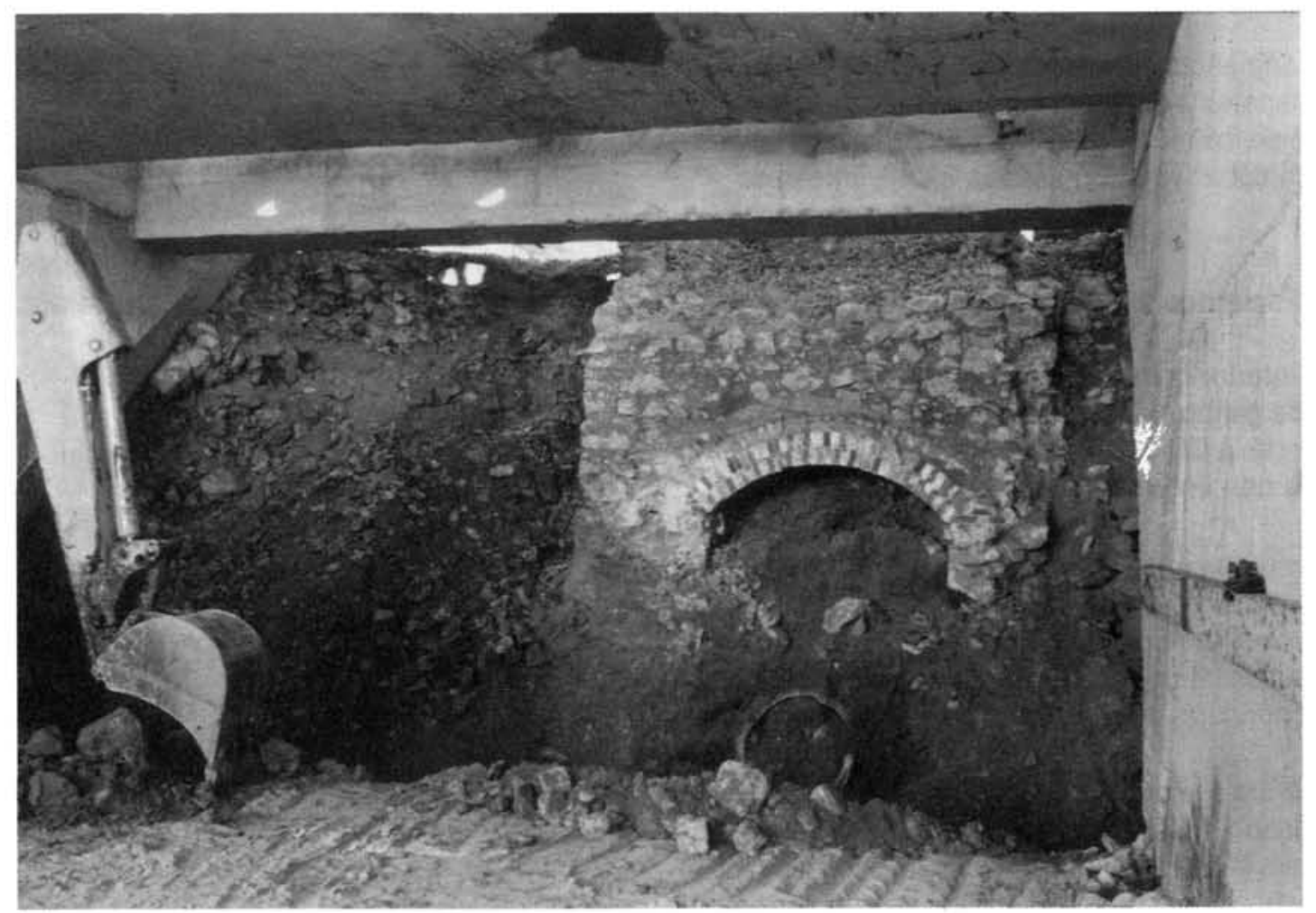

Foto 12

LINEA TARRAGONA - SAN VICENTE P. K. $2 / 678$

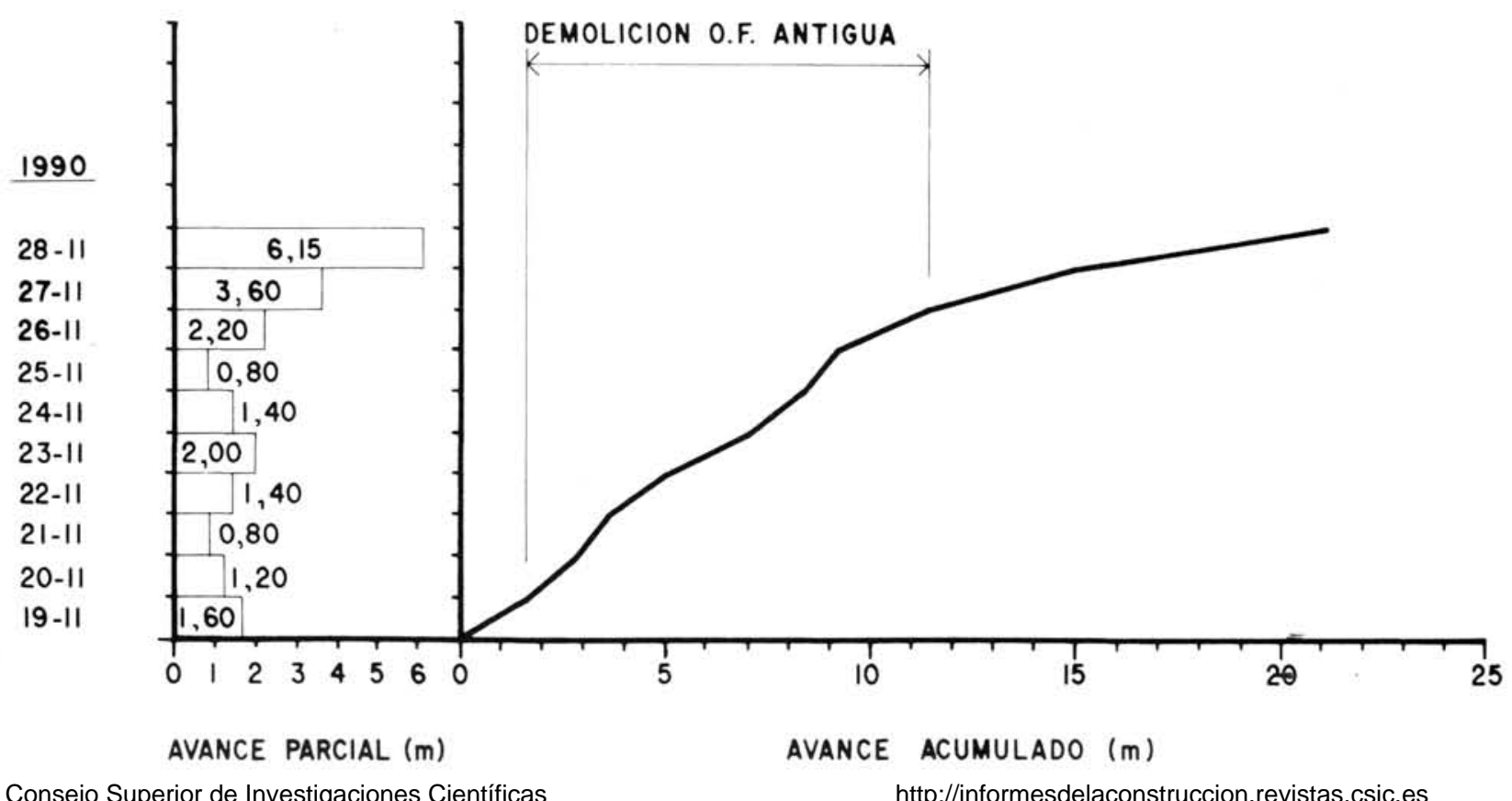

(c) Consejo Superior de Investigaciones Científicas Licencia Creative Commons 3.0 España (by-nc) 


\section{Punto kilométrico 5/419}

Este paso de gálibo restringido se ha construido para dar acceso al Camping de Las Palmeras desde la $\mathrm{CN}-340$, con una anchura interior de $6,50 \mathrm{~m}$ y gálibo restringido de $3,70 \mathrm{~m}$. La plataforma superior es de $13 \mathrm{~m}$ para doble via (Foto 13).

En la ejecución de la obra se presentaron, al mismo tiempo, tres de los inconvenientes más importantes pa. ra la realización de pasos por empuje oleodinámico: nivel freático alto, arenas sueltas y roca dura.

La via está situada sobre arenas finas de playa, con un espesor medio de $3 \mathrm{~m}$, que descansan sobre un substrato calizo. El nivel freático se encuentra a $-2,50 \mathrm{~m}$ bajo la vía, coincidiendo con el nivel del mar.

Aunque suponia un mayor volumen de excavación en roca, la prefabricación de la estructura se realizó en lado montaña, ya que se conseguia de esta forma un recinto de trabajo más estanco y se aprovechaba la roca para el apoyo del muro de reacción. Incluso con esta disposición, fue preciso tener en funcionamiento dos bombas de achique, de gran caudal, todo el tiempo que duró la construcción de la estructura, debido a la afluencia continua de agua por las fracturas de la roca (Foto 14).

Para evitar la afluencia de agua por el frente de excavación, durante la traslación de la estructura, se rebajó el nivel freático en toda la zona a ocupar por el sistema de Well-point (Foto 15), y se continuó achicando agua con las bombas en el recinto de construcción. De no haberse utilizado dicho sistema, además de tener la zona de trabajo inundada, la filtración de agua habria producido importantes arrastres de arena poniendo en peligro el servicio de la via.

Al rebajar la lámina de agua, las arenas adquirian una compacidad bastante aceptable por tensión superficial del agua residual. No obstante, las vibraciones del martillo que realizaba la excavación de la roca de base produjeron algunos desprendimientos laterales de la arena, obligando a ampliar la zona de sustentación de la via mediante vigas de maniobra situadas fuera de la estructura y a ambos lados de la misma (Foto 16). Para esta operación se detuvo el avance durante el quinto dia, continuando con normalidad al dia siguiente.

(C) Consejo Superior de Investigaciones Científicas Licencia Creative Commons 3.0 España (by-nc)

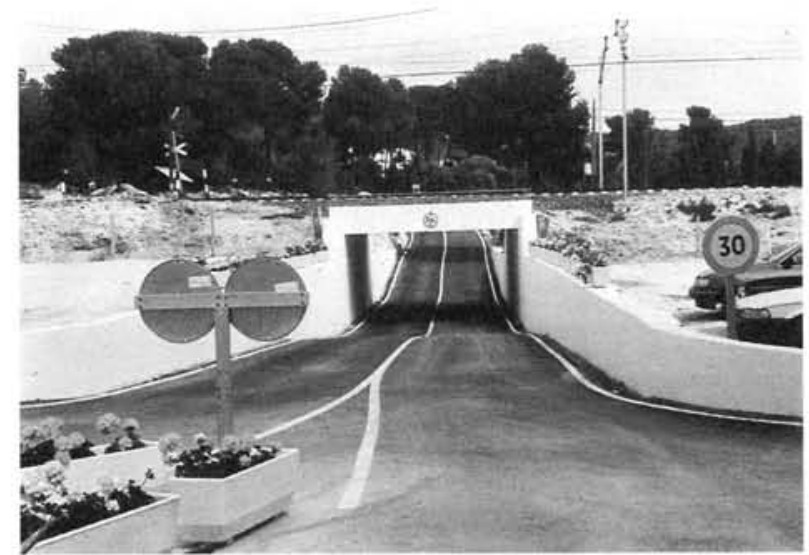

Foto 13

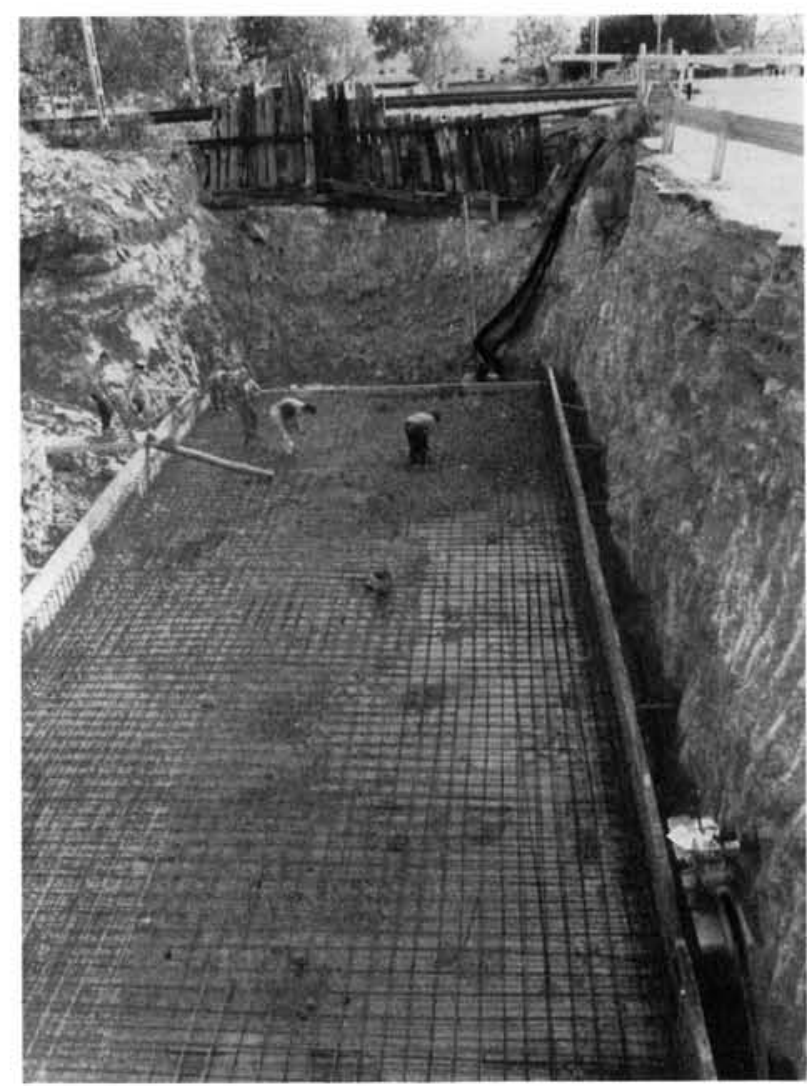

Foto 14

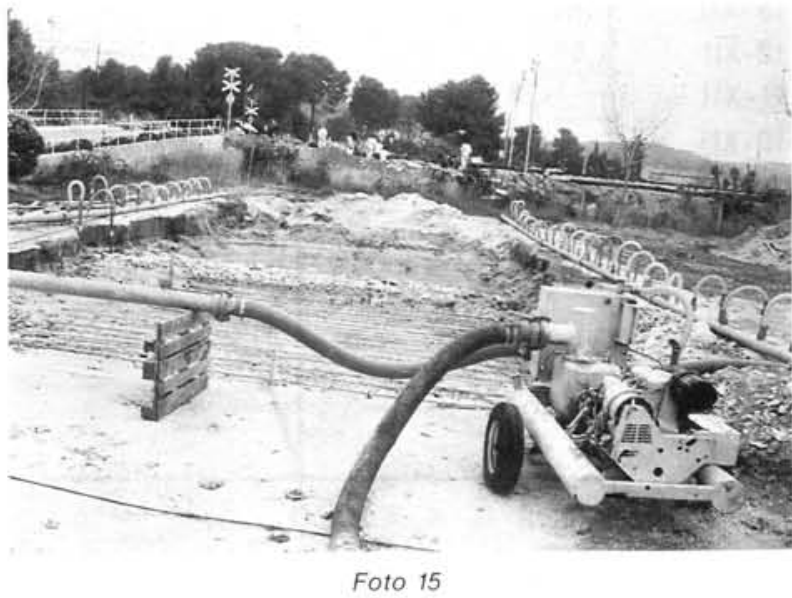

http://informesdelaconstruccion.revistas.csic.es 


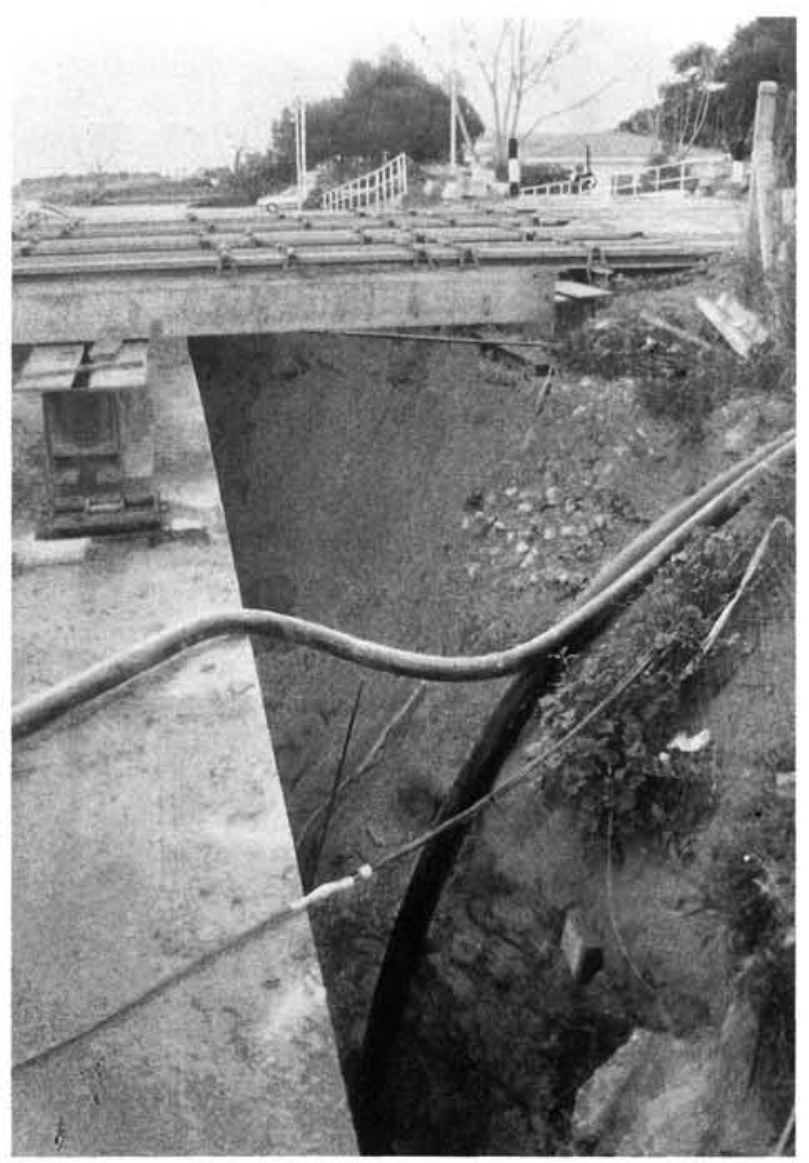

Foto 16

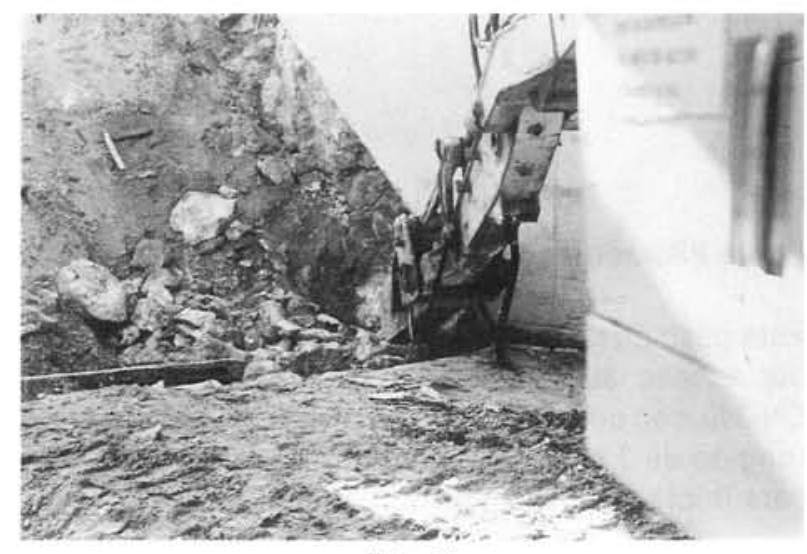

Foto 17

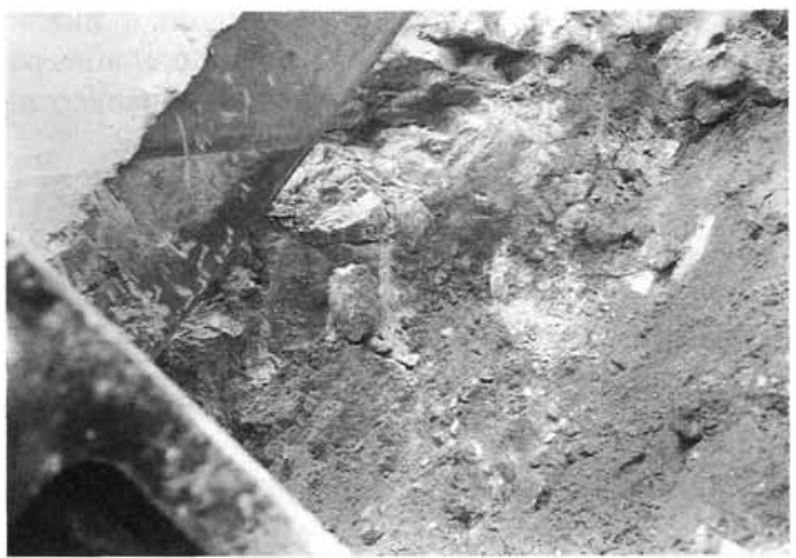

Foto 18

LINEA TARRAGONA - SAN VICENTE P. K. $5 / 419$

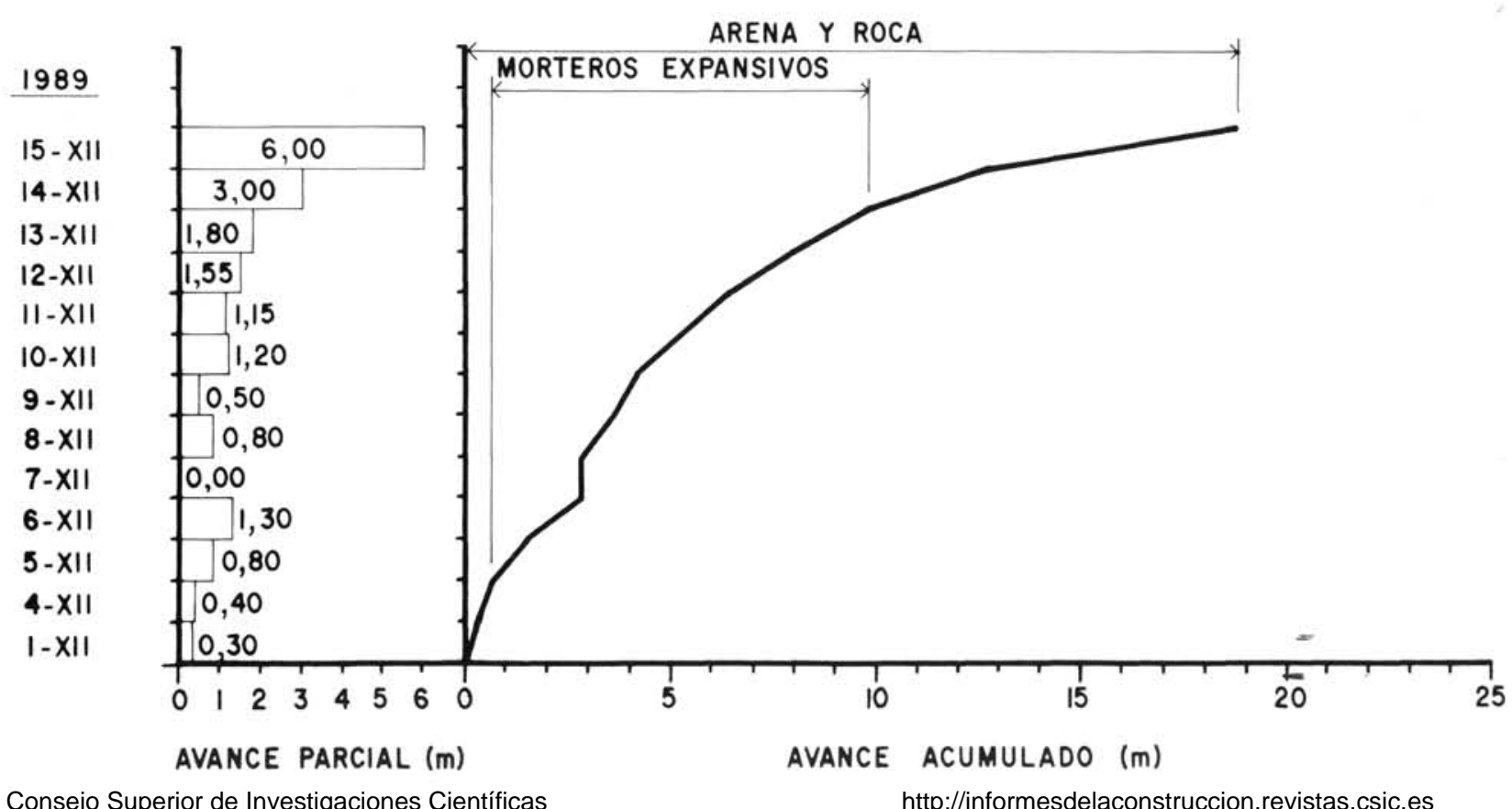

(c) Consejo Superior de Investigaciones Científicas

http://informesdelaconstruccion.revistas.csic.es 
Dado que la maquinaria que se pudo utilizar para la excavación de la roca era de dimensiones reducidas, limitadas por las propias de la estructura, el rendimiento de los martillos rompedores era escaso, estando restringida también su maniobrabilidad (Foto 17). Para facilitar la labor de éstos, se usaron morteros expansivos que realizaban una fracturación primaria de la roca. Estos morteros se introducian en taladros perforados previamente, y al final de la jornada, ejerciendo su acción durante la noche (Foto 18).

Pese a todos los inconvenientes descritos, la traslación se concluyó en 13 dias para una longitud total de 18,80 metros, resultando un avance medio de $1,45 \mathrm{~m} / \mathrm{dia}$. En el diagrama de avance se aprecia el escaso rendimiento obtenido trabajando sólo con martillos en los dos primeros días $(0,35 \mathrm{~m} / \mathrm{dia})$ y el aumento obtenido con la utilización de morteros expansivos a partir del tercer dia, obteniendo una media de 1,14 m/dia.

La experiencia de estas tres obras nos lleva a la conclusión de que, salvo casos extremos, las caracteristicas del terreno no son un condicionante restrictivo para la construcción de pasos inferiores por el sistema de empuje oleodinámico, puesto que se puede combinar con todos los métodos conocidos de excavación, desecación, contención y consolidación del terreno.

En todo caso se precisará un tiempo algo mayor para realizar la traslación de la estructura y la disposición de otros medios auxiliares, pero sigue existiendo una gran diferencia en el tiempo de afección al ferrocarril respecto de los métodos tradicionales, y posiblemente sean necesarios los mismos medios auxiliares en ambos casos.

Los autores expresan su agradecimiento a la Empresa ATROS, S. A. realizadora de los empujes por su colaboración y entusiasmo; y a la Empresa Cubiertas y MZOV por el apoyo e ilusión que derrocharon en la realización de las obras de paso inferior; y, como no, a RENFE por la confianza y facilidades que nos dieron para el desarrollo de este nuevo sistema constructivo que tanto facilita nuestra labor de eliminar pasos a nivel.

\title{
publicación del ICCET/CSIC
}
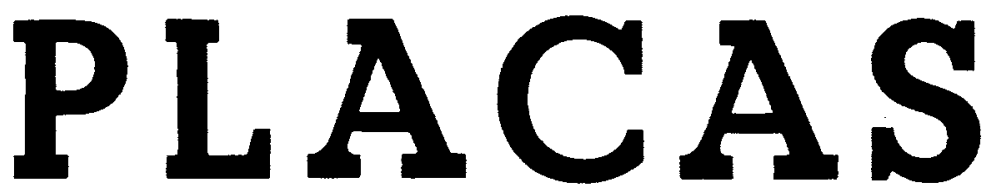

\author{
K. Stiglut g H. Wippel \\ Drs. Ingenieros \\ Traducción de Juan Batanero \\ Dr. Ingeniero de Caminos \\ con la colaboración de \\ Francisco Morán \\ Ingeniero de Caminos
}

\begin{abstract}
Este libro, cuidadosa y magníficamente editado, reúne, quizás, la más completa colección conocida de tablas para placas, por los numerosos casos de vinculación y de carga estudiados y por la abundancia de relaciones de dimensión y de datos ofrecidos, que cubren prácticamente todo el campo de las losas en edificación. Permite desarrollar, con comodidad, rapidez y una aproximación suficiente, los cálculos de dimensionamiento y comprobación, obviando las dificultades que como es sabido, presenta el desarrollo numérico de los métodos de cálculo de estos elementos, evitando enojosas operaciones.

Trata la obra sobre "Zonas de Placas", "Placas sobre apoyos puntuales", "Placas apoyadas en dos, tres y cuatro bordes" y "Placas apoyadas elásticamente", tipos que en la actualidad disponian de una documentación, incompleta o nula, para la determinación de esfuerzos. Los corrimientos de la placa, como valores previos para la determinación de los momentos, han sido obtenidos por medio del Cálculo de Diferencias, método que se ha comprobado como suficientemente satisfactorio, aún en su forma simple, aplicado con un cierto control.
\end{abstract}

Un volumen encuadernado en tela, de $30,5 \times 23,5 \mathrm{~cm}$, compuesto de 92 págs. Madrid, 1968 . 